\title{
Adult-onset autosomal recessive ataxia with thalamic lesions in a Finnish family
}

\author{
M. Rantamäki, MD; R. Krahe, PhD; A. Paetau, MD, PhD; B. Cormand, PhD; \\ I. Mononen, $\mathrm{MD}$; and $\mathrm{B}$. Udd, $\mathrm{MD}, \mathrm{PhD}$
}

\begin{abstract}
Article abstract-Objective: To describe an unusual kindred with adult-onset ataxia and thalamic lesions detected by brain MRI. Methods: The authors characterized clinical, laboratory, and pathologic features of the disease and sought linkage to previously recognized ataxia loci. Results: Two sisters and a brother developed progressive ataxia, dysarthria, mild cognitive impairment, and sensorimotor neuropathy at age 30, combined with epilepsy in one sibling. MRI showed symmetric thalamic lesions, changes in brainstem gray matter, and white matter changes in the cerebellum. Autopsy in one of the patients revealed neuronal degeneration with a peculiar vacuolar change in thalamus, probably representing transsynaptic degeneration in response to deafferentation. Neuronal and secondary tract degeneration was observed in the spinal cord, cerebellum, and brainstem suggesting a spinocerebellar degeneration. The disorder appears to be transmitted as an autosomal recessive trait. Genetic and sequence analysis of the FRDA gene and comprehensive laboratory examinations excluded Friedreich's ataxia and other similar recessive diseases. Conclusion: Adult-onset recessive ataxia with bilateral thalamic lesions in this family may represent a distinct hereditary spinocerebellar ataxia.
\end{abstract}

NEUROLOGY 2001;57:1043-1049

Late-onset autosomal recessive ataxia is very rare; onset before age 25 used to be a principal feature of Friedreich's ataxia (FRDA1) in the classification by Harding. ${ }^{1}$ The detection of a major mutation in the frataxin (FRDA) gene, an unstable (GAA) trinucleotide repeat in intron 1 , allows for rapid and accurate molecular diagnosis. ${ }^{2}$ Recently, patients with adult-onset FRDA1 have also been identified by genetic testing of FRDA. ${ }^{3}$

The other well-defined recessive ataxias typically have an early onset. Disease-causing mutations have been identified in specific genes for ataxia with vitamin E deficiency (AVED) ${ }^{4}$ and ataxia telangiectasia $(\mathrm{A}-\mathrm{T}),{ }^{5}$ both of which are mainly childhood disorders. An autosomal recessive form of childhood-onset ataxia with spasticity (ARSACS), first reported in French-Canadian families from the CharlevoixSaguenay region of Quebec, has been mapped to $13 \mathrm{q} 11^{6}$ with an allelic syndrome recently reported from Tunisia. ${ }^{7}$ Early-onset cerebellar ataxia (EOCA) is genetically heterogenous, and the genetic defects underlying most forms are unknown. ${ }^{8}$ However,

Additional material related to this article can be found on the Neurology Web site. Go to www.neurology.org and scroll down the Table of Contents for the September 25 issue to find the title link for this article. some patients with EOCA have mutations in the FRDA gene and are diagnosed with Friedreich's ataxia with retained reflexes. ${ }^{9}$ One form of EOCA, infantile-onset recessive ataxia, has been mapped to 10q23.3-10q24.1. ${ }^{10}$ Other early-onset forms of recessive ataxia have been reported, each occurring with one of the following clinical features: hypogonadism, congenital or childhood deafness, optic atrophy, pigmentary retinopathy, extrapyramidal symptoms, cataracts, or mental retardation. ${ }^{1}$

Here we describe a kindred with five sibs, three of them affected by progressive ataxia starting at age 30. Clinical, biochemical, and molecular genetic investigations have ruled out FRDA1, ARSACS, and other known ataxias causing a similar symptomatology.

Patients and methods. Two sons were healthy, and one 45-year-old son and two daughters aged 36 and 42 years were affected (figure 1). The youngest patient died at the end of the study. Preliminary autopsy findings are included in this report, and a complete study will be published separately. Both parents and all four grandparents were born in the same rural area in Karelia, near the Finnish-Russian border.

The parents were examined by two neurologists (M.R. and B.U.); both were healthy. No other family members

From the Department of Neurology and the Department of Physical Medicine and Rehabilitation (Dr. Rantamäki), Seinäjoki Central Hospital, Seinäjoki, Finland; the Division of Human Cancer Genetics (Dr. Krahe), Comprehensive Cancer Center, Ohio State University, Columbus; the Department of Molecular Genetics (Dr. Krahe), Folkhälsan Institute of Genetics, University of Helsinki, Finland; the Department of Pathology (Dr. Paetau), University of Helsinki, Finland; the Department of Molecular Genetics (Dr. Cormand), University of Barcelona, Spain; the Department of Clinical Chemistry and Hematology (Dr. Mononen), Turku University Central Hospital, Finland; the Department of Neurology (Dr. Udd), Vasa Central Hospital, Finland; and Tampere University Hospital (Dr. Udd), Finland.

Supported by a grant from the Medical Research Fund of Tampere University Hospital. R.K. was supported in part by grant P30-CA16058 from the National Cancer Institute, Bethesda, MD. B.C. is a recipient of a grant from the Ministerío de Educación y Cultura, Spain. Part of the research was conducted at the Folkhälsan Institute of Genetics in Helsinki.

Received May 15, 2000. Accepted in final form May 24, 2001.

Address correspondence and reprint requests to Dr. Maria Rantamäki, Seinäjoki Central Hospital, 60220 Seinäjoki, Finland; e-mail: maria.rantamaki@pp.fimnet.fi 


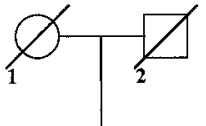

II
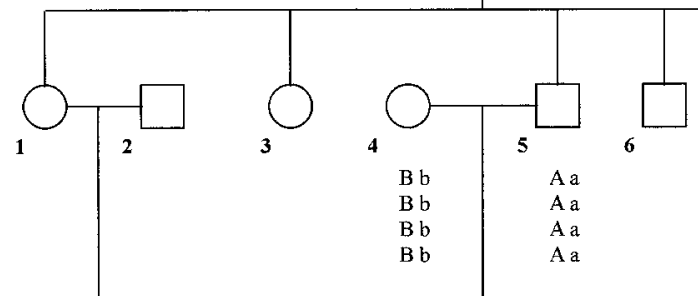

\begin{tabular}{ll|ll}
3 & 4 & 5 & 6
\end{tabular}

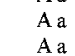

$\mathrm{A}$ a $\mathrm{a}$ $\mathrm{Bb} \quad \mathrm{Aa}$

III
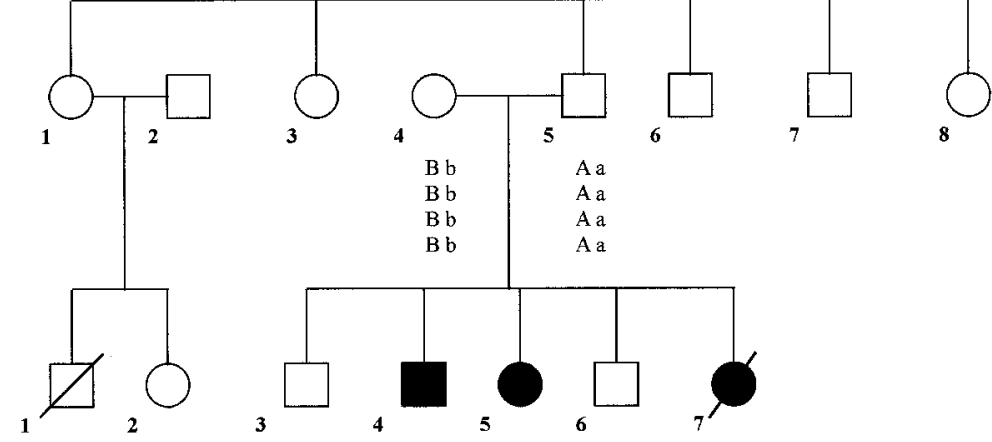

FRDA markers A a B b

$\begin{array}{lllll}\text { D9S741: } & 208 & 197 & 189 & 203\end{array}$

D9S1118: $\quad \begin{array}{lllll}168 & 164 & 156 & 172\end{array}$

D9S301:

D9S1122: $\quad \begin{array}{lllll}192 & 192 & 200 & 196\end{array}$

$\begin{array}{lrrrr}\text { ARSACS markers } & \text { A } & \text { a } & \text { B } & \text { b } \\ \text { D13\$175: } & 107 & 109 & 113 & 109\end{array}$

D13S787: $\quad 256 \quad 260256 \quad 260$

D13S217: $\quad \begin{array}{lllll}258 & 256 & 260 & 260\end{array}$

$\begin{array}{lllll}\text { D13S171 : } & 189 & 199 & 189 & 189\end{array}$

3

4

$\begin{array}{lll}A \text { B } & \text { A b } & \text { A B } \\ \text { A B } & \text { A b } & \text { A B } \\ \text { A B } & \text { A b } & \text { A B }\end{array}$

A B A b $\quad A$ b

$\begin{array}{lll}\text { A } & \text { B } & \text { A B } \\ \text { A } & \text { B } & \text { A B } \\ \text { A } & \text { B } & \text { A B }\end{array}$

A B A B
Figure 1. Family pedigree shows the haplotypes for FRDA and ARSACS loci segregating with parents and affected members. Filled symbols indicate affected family members. were affected by any similar disease. Two cousins had had neurologic problems (III-1 and III-2). One exhibited severe psychiatric symptoms that started at age 32 with alcoholism. He died suddenly at age 42 , presumably from an epileptic seizure. No macroscopic changes in the brain were reported on autopsy. His 45-year-old sister had congenital cerebral palsy without subsequent progressive neurologic problems.

One of the affected individuals (III-4) refused further clinical examinations. However, he agreed to clinical examination by two neurologists (M.R. and B.U.). The two other patients (III-7, the proband, and III-5) underwent laboratory examinations of blood, urine, CSF, and fibroblast samples. Laboratory tests for lysosomal storage diseases included urinary screening for mucopolysaccharides and oligosaccharides by thin layer chromatography, and determination of the enzyme activity of arylsulfatase A, galactocerebrosidase, $\beta$-galactosidase, glucocerebrosidase, hexosaminidase $\mathrm{A}$ and $\mathrm{B}$, and $\alpha$-neuraminidase in fibroblasts. CT and MRI of the brain were performed. Neurophysiologic investigation included motor and antidromically determined sensory nerve conduction measurements using surface electrodes, sensory threshold measurements, electromyography (EMG), tibial and median nerve somatosensoryevoked potentials (SEP), brainstem auditory-evoked potentials, and reversal pattern visual-evoked potential (VEP), together with flash VEP, electroretinography and blink reflex, and EEG. Muscle and sural nerve biopsies and bone marrow aspiration were performed on Patient III-7. After her sudden death, autopsy and neuropathologic studies were conducted. Other examinations included neuropsychologic tests and ophthalmologic examinations, electrocardiography, thoracic and long-bone x-rays, abdominal ultrasonography, and a set of tests to examine functions of the autonomous nervous system.

DNA isolation. Seven members of the family were included in the genetic study (figure 1). Genomic DNA was isolated from venous blood or lymphoblastoid cell lines from all consenting individuals using standard methods.
All the samples were drawn in accordance with the Helsinki Declaration.

Genotyping. To determine linkage in the FRDA1 and ARSACS $^{6,7,11}$ regions in chromosome 9q13 and 13q11, we typed four microsatellite markers ${ }^{12}$ flanking each candidate gene: D9S741 (42.7 cM), D19S1118 (58.3 cM), D9S301 (66.3 cM), D9S1122 (75.9 cM), D13S175 (5.03 cM), D13S787 (8.87 cM), D13S217 (17.21 cM), and D13S171 $(25.08 \mathrm{cM})$. Genomic DNA samples were analyzed by PCR amplification. The forward primers were modified at the $5^{\prime}$ end with a FAM, TET, or HEX fluorescent label. The PCR reactions were performed under the following conditions: 50 ng genomic DNA, $1 \times$ Perkin-Elmer PCR buffer (Foster City, CA), $130 \mu \mathrm{M}$ dNTP, 5 pmols of both the forward and the reverse primer, and 0.75 U Ampli Taq Gold ${ }^{\mathrm{TM}}$ polymerase (Perkin-Elmer) in a final volume of $15 \mu \mathrm{L}$. The cycling conditions were $94{ }^{\circ} \mathrm{C}$ for 10 minutes for one cycle, $94{ }^{\circ} \mathrm{C}$ for 30 seconds, $55{ }^{\circ} \mathrm{C}$ for 1 minute 15 seconds, and $72{ }^{\circ} \mathrm{C}$ for 1 minute, for 30 cycles (FAM and TET markers) or 35 cycles (HEX markers), and $72{ }^{\circ} \mathrm{C}$ for 10 minutes. Amplified products were separated by electrophoresis on $4.25 \%$ polyacrylamide-6 M urea gel using a 377 DNA sequencer (Perkin-Elmer/Applied Biosystems, Weiterstadt, Germany), and the results were processed with Genescan ${ }^{\mathrm{TM}}$ (version 2.0.2) and Genotyper ${ }^{\mathrm{TM}}$ (version 1.1) software (Perkin-Elmer/Applied Biosystems).

Mutation analyses. Detection of the $(\mathrm{GAA})_{\mathrm{n}}$ expansion in intron 1 of the FRDA gene was performed as described, ${ }^{2}$ except that the $5^{\prime}$ and $3^{\prime}$ primers were tailed with M13forward (5'-TGTAAAACGACGGCCAGT-3') and M13reverse (5'-CAGGAAACAGCTATGACC-3') tails for direct sequencing in both directions. PCR fragments were purified using the Exonuclease I/Shrimp Alkaline Phosphatase PCR Product Sequencing Kit (USB-Amersham Life Science, UK). Two $\mu$ l of the PCR products was sequenced using the BigDye-Terminator AmpliTaq FS Cycle Sequencing Kit (Perkin-Elmer/Applied Biosystems), and results were analyzed with Sequence Navigator software (Perkin-Elmer/Applied Biosystems). 
Table Clinical findings of affected individuals in the pedigree

\begin{tabular}{|c|c|c|c|}
\hline Finding & Patient III-7 & Patient III-5 & Patient III-4 \\
\hline Ataxia of limbs, gait, and stance & +++ & +++ & +++ \\
\hline Dysarthria & ++ & ++ & +++ \\
\hline Dysphagia & + & + & - \\
\hline Nystagmus and jerky pursuit eye movements & + & + & + \\
\hline \multicolumn{4}{|l|}{ Tendon reflexes } \\
\hline Upper limbs & Normal & Normal & Decreased \\
\hline Knee and ankle & Decreased & Absent & Absent \\
\hline Babinski sign & $+/+$ & $-1-$ & $-1-$ \\
\hline
\end{tabular}

+ Finding present; ++ prominent finding; +++ severe finding; - finding absent.

PCR was used for the amplification of $(\mathrm{CAG})_{\mathrm{n}}$ repeats, and polyacrylamide gel electrophoresis for estimation of repeat numbers in the autosomal dominant SCA types 1 , $2,3,6,7,8,10$, and 12 and dentatorubral-pallidolluysian atrophy (DRPLA) according to standard techniques previously described. ${ }^{13-21}$ DNA mutation analyses of mitochondrial point mutations at positions 8344, 3243, and 8993 of the mtDNA associated with myoclonus epilepsy with ragged-red fibers (MERRF), mitochondrial encephalomyopathy-lactic acidosis, stroke-like symptoms (MELAS), neuropathy, ataxia with retinitis pigmentosa (NARP), and mitochondrial deletion screening were carried out from muscle samples of Patient III-7 according to methods described elsewhere. ${ }^{22}$

Case reports. Patient III-7. Her developmental milestones were normal. Neurologic symptoms started at age 29 with increasing difficulties in walking and balance. Her husband noticed decreased initiative and ability to concentrate. At age 30, severe gait ataxia was observed. MS was suspected, but CSF showed no signs of demyelinating disease. The patient had repeated grand-mal-type epileptic seizures, which were resistant to anticonvulsive medication. Dysarthria started after age 30, and the patient also reported slight dysphagia. In later years, the walking distance with a walker decreased to a few hundred meters, but the patient continued to be ambulatory until her sudden death at age 36 , presumed to be caused by an epileptic seizure.

On clinical examination at age 36 , the patient exhibited a grossly ataxic, wide-based gait while leaning on a walker. She had mild tremor of the head. Muscle strength and tone in the upper and lower extremities were normal, and there was no muscular atrophy. Finger-to-nose test was dysmetric, and diadochokinesia was clumsy. She had decreased fine motor movement in the fingers. Lower limbs showed marked dysmetria. Vibration and position senses were impaired, whereas her superficial sense was intact. Patellar and ankle reflexes were barely elicited, and Babinski sign was positive. Eye movements were full with gaze-evoked nystagmus and jerky pursuit movements. She had diplopia when changing direction of gaze to the left. Her speech was mildly slurred. Mental alertness, interaction, orientation, and perception were good, but short-term memory was inaccurate.

Patient III-5. Early development and adolescence were normal. At approximately age 30, the patient noticed clumsiness in her legs while walking down stairs. Neurologic examination at age 36 showed pronounced ataxia. She also had mild dysphagia, and stress incontinence started at age 37. At age 42 she was using a walker, and her gait was ataxic, wide-based, but fairly rapid.

Patient III-4. Childhood development was normal. The patient performed military service at age 21 without problems. At approximately age 30, he started to show lack of initiative, tiredness, social withdrawal, slight balance problems, and clumsiness on walking. At age 35, he was referred to a mental health center. Lack of concentration and withdrawal were considered to suggest mental illness. Antipsychotics increased difficulty of movement, and he stopped taking them. The patient refused further neurologic examinations. Difficulty in walking worsened over time, his speech was slurred, and his hands were clumsy. The patient was aware of slight memory problems. He also had pollaciuria. Upon neurologic examination at age 44 he could stand without support, and walking was grossly ataxic.

Clinical findings are summarized in table 1.

Results. Brain imaging findings. The proband (III-7) underwent brain MRI three times, at ages 32, 33, and 35 (figure 2, A through B). Main findings included increased signal intensity lesions in T2-weighted series bilaterally in the thalamus and in the upper part of the medulla oblongata at the level of inferior olivary nuclei, primarily in the gray matter. In addition, bilateral high-intensity signals were seen in the cerebellar white matter. As with demyelination, these lesions were hypointensive in T1-weighted series. A small frontal cortical lesion observed on the first MRI was not visible in subsequent studies, but other findings persisted. No contrast enhancement was found. There 

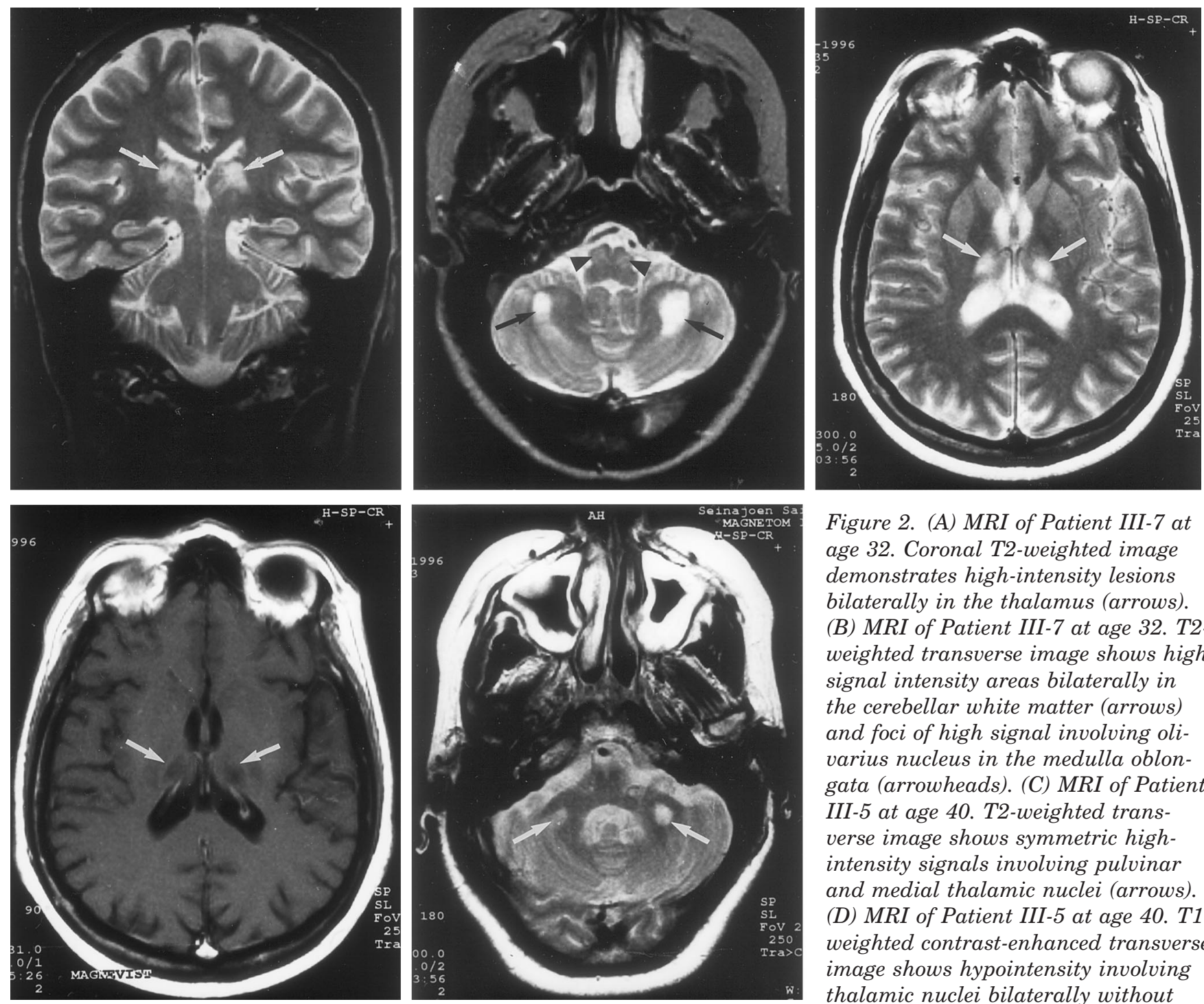

Figure 2. (A) MRI of Patient III-7 at age 32. Coronal T2-weighted image demonstrates high-intensity lesions bilaterally in the thalamus (arrows). (B) MRI of Patient III-7 at age 32. T2weighted transverse image shows high signal intensity areas bilaterally in the cerebellar white matter (arrows) and foci of high signal involving olivarius nucleus in the medulla oblongata (arrowheads). (C) MRI of Patient III-5 at age 40. T2-weighted transverse image shows symmetric highintensity signals involving pulvinar and medial thalamic nuclei (arrows). (D) MRI of Patient III-5 at age 40. T1weighted contrast-enhanced transverse image shows hypointensity involving thalamic nuclei bilaterally without contrast enhancement (arrows). (E) MRI of Patient III-5 at age 40. T2-weighted transverse image demonstrates increased signal areas in middle cerebellar peduncles (arrows) and enlargement of fourth ventricle and pontine cysterns.

were minor infra- and supratentorial atrophic changes. Brain CT performed at age 32 was normal.

Patient III-5 underwent one brain MRI at age 40 (see figure 2, C through E). Symmetric thalamic and cerebellar hyperintensity signal abnormalities similar to those observed in her sister were detected in T2-weighted series, and these lesions were hypointensive in T1-weighted images. She had minor infra- and supratentorial atrophy. Symmetric low density areas in the thalamus and cerebellar white matter were also observed on brain CT performed at age 37 .

Laboratory findings. Detailed blood, urine, and CSF tests were performed for the proband (III-7) and Patient III-5 without abnormal findings. Complete blood counts were normal. No acanthocytosis could be demonstrated in peripheral blood smears. Results relating to fasting glucose, glucose tolerance, electrolytes, creatinine, calcium, vitamin $\mathrm{E}$, vitamin $\mathrm{B}_{12}$, folic acid, creatine kinase, lactate dehydrogenase, serum electrophoresis, serum immunoglobulins, $\alpha$-fetoprotein, very long chain fatty acids, phytanic acid, and cholestanol were normal. No significant abnormalities were demonstrated in liver and thyroid 1046 NEUROLOGY 57 September (2 of 2) 2001 function tests, cholesterol and triglyceride levels, copper and ceruloplasmin values, or lactate and pyruvate levels. Blood ammonia and urinary organic and amino acids, CSF protein level, cell count, and glucose, lactate, and IgG indexes were all normal, as were laboratory tests for lysosomal disorders.

Results of basic cardiologic examinations, ECG, and chest x-rays were normal in Patients III-7 and III-5. Echocardiography in Patient III-7 showed minor mitral valve insufficiency but no signs of cardiomyopathy. Findings on abdominal ultrasonography and x-rays of long bones were also normal.

Neurophysiologic features. Sensory and motor conduction velocities were slightly reduced in Patients III-7 and III-5, and sensory action potentials in peroneal and sural nerves were absent or had markedly decreased amplitudes. The findings were consistent with a mainly sensory axonal neuropathy (findings summarized in table 2, available at www.neurology.org). On sensory threshold testing, Patient III-7 exhibited impaired vibration sense. Cold and heat thresholds were also abnormal, suggesting thin-fiber neuropathy. 
Results of SEP tests of both patients demonstrated similar findings. Responses were diffusely delayed at the spinal level and missing or decreased at the cortical level, suggesting a central disorder in the somatosensory pathway. The central conduction time T12-P37 of the tibial SEP recorded from Patient III-7 was normal (18.3 msec on both sides). Tibial SEP P37 in Patient III-5 was remarkably prolonged (49.2 $\mathrm{msec}$ on the right side/49.3 $\mathrm{msec}$ on the left side), but because of missing spinal responses the exact level of the latency delay remains uncertain. Brainstem auditory-evoked potential stimulation of the right ear in Patient III-7 showed inconsistent brainstem responses. On the left, latencies were slightly prolonged, suggesting a disorder in the region of the pons. Latency of wave I was $1.7 \mathrm{msec}$, interpeak latency I-III $2.2 \mathrm{msec}$, and interpeak latency I-V $4.3 \mathrm{msec}$ on the left side. Reversal pattern VEP, flash VEP, blink reflex, and electroretinograms were normal. EEG of Patient III-7 at age 33 revealed moderate diffuse background slowing. Autonomic function tests of Patient III-7 showed findings of the cardiovascular system compatible with mild autonomic dysfunction.

Specific studies. Ophthalmologic examination of $\mathrm{Pa}-$ tients III-7 and III-5 revealed no retinopathy, optic nerve atrophy, or cataracts.

Extensive neuropsychologic examinations of Patient III-7 and III-5 showed cognitive and behavioral changes. The overall cognitive processing was at low-medium level: the full scale IQ of Patient III-7 was 88 (Wechsler Adult Intelligence Scale-Revised) and of Patient III-5 86 (Wechsler Adult Intelligence Scale), although the primary IQ level of both patients was supposed to be normal. Verbal and visual memory measured by Wechsler Memory Scale and visuomotor functions were impaired. Patient III-7 also had mild anomia and flattening of affect, and both patients had some loss of insight.

Morphologic studies and autopsy findings. Minor, nonspecific changes without significant neurogenic atrophy were observed on light and electron microscopic examination of muscle biopsy specimens, and there were no findings suggesting storage or mitochondrial diseases. Light and electron microscopy of distal sural nerve showed marked decrease of large myelinated fibers and some clusters of small regenerating fibers. No demyelination was observed. The pathologic findings were consistent with axonal neuropathy. No pathologic findings indicating storage disease were detected on bone marrow examination.

On autopsy the cerebral hemispheres were preserved, but degenerative pathology was observed in the thalamus, brainstem, cerebellum, and spinal cord. Slight degeneration and atrophy were seen in the dorsal root ganglia. The spinal nerve roots, predominantly the posterior, showed some fiber depletion. The posterior columns (especially the gracile), the posterior spinocerebellar tracts (to a lesser extent), and the corticospinal tracts (to an even milder extent) were atrophic. The dorsal nucleus (Clarke's column) showed a clear neuronal loss, which was not the case in the autonomic intermediolateral columns. In the cerebellum, the cortex was quite well preserved, except for a slight and patchy dropout of Purkinje cells. The most severe changes in the cerebellum were seen in the dentate: subtotal depletion of neurons, gliosis, and completely fiberdepleted hilus. In medulla oblongata, a similarly severe degeneration of the inferior olives was seen. The caudal pontine basis was slightly atrophic, as were the corticospinal tracts, pontine, mesencephalic tegmentum, and periaqueductal gray matter. Inferior and superior cerebellar peduncles were atrophic, but middle cerebellar peduncles were only slightly atrophic. Substantia nigra was atrophic, especially at its pars lateralis. Some neuronal degeneration in the pulvinar, and especially in the dorsomedial thalamic nuclei, accompanied by a peculiar vacuolar change, was observed, and this morphologic change most probably represents a transsynaptic deafferentationrelated atrophy. Basal ganglia, hippocampi, and white matter were well preserved. There were some focal laminar necrosis remnants in the cerebral cortex, but morphology was mostly normal. No signs of neuronal storage could be observed.

Genetic analyses. Three members of the family presented here had the disease, whereas no symptoms were observed in the parents or the other two healthy siblings (figure 1). Both sexes were affected without major variations in the phenotype. Genealogic studies revealed no links among parents over the past 150 years, but the fact that they were born in the same rural community increases the probability of distant common ancestry. An autosomal dominant genetic defect with incomplete penetrance or maternal inheritance were considered unlikely because of the absence of phenotypic variation, including age at onset, and no signs of disease, even to a lesser extent, in the healthy family members. Taken together, these findings are most consistent with an autosomal recessive inheritance pattern.

DNA analyses for the major mutations in autosomal dominant SCA types 1, 2, 3, 6, 7, 8, 10, and 12, DRPLA, mitochondrial deletions, and point mutations associated with MERRF, MELAS, and NARP were all negative.

In addition, we considered linkage to the two autosomal recessive ataxia loci currently characterized, FRDA in $9 q 13$ and $S A C S$ in 13q11. Although all three patients were heterozygous for the four polymorphic markers flanking the FRDA locus, the two patients (III-7 and III-5) tested for the FRDA1-associated (GAA) ${ }_{n}$ expansion were homozygous for the same (GAA) ${ }_{10}$ allele. This suggested the possibility of a homozygous mutation in FRDA other than the $(\text { GAA })_{n}$ expansion. To exclude such a mutation, we sequenced the entire region, coding and flanking intronic sequences. No mutations were identified. Haplotypes constructed with four polymorphic markers in the ARSACS and FRDA1 regions show that both parental chromosomes are inherited among the patients for both disease loci (see figure 1), thus excluding linkage.

Discussion. Here we describe a family with progressive adult-onset spinocerebellar ataxia in three affected siblings. Clinical findings include gait ataxia, dysarthria, nystagmus, peripheral neuropathy, mild impairment in cognition, and, in one patient, epileptic seizures. Bilateral symmetric thalamic lesions constitute a very specific finding on brain MRI. Other MRI findings are cerebellar white matter changes and mild infra- and supratentorial atrophy. The presentation of the disorder in the family is most compatible with an autosomal recessive mode of inheritance.

Some features may suggest a diagnosis of FRDA. ${ }^{23}$ 
These include ataxia, dysarthria, loss of deep sensation, and absent or mild cerebellar atrophy. FRDA1 may also be associated with cardiomyopathy or diabetes, but these were not present in this family. All patients had cognitive impairment and one had epilepsy, which are not features of FRDA1. Unique MRI and morphologic findings of bilateral thalamic lesions have not been reported in FRDA1 or any other recessive ataxia. An FRDA1-associated mutant $(\text { GAA })_{n}$ expansion in FRDA was excluded as the underlying mutation.

Gene sequencing and linkage analysis exclude other mutations of the FRDA gene. We also considered linkage to the other characterized recessive ataxia locus, SACS, in 13q11. Nonidentical sharing of parental chromosomes in the patients shown by segregation of haplotypes excluded linkage to the ARSACS region.

Recessive ataxia caused by abnormal vitamin $\mathrm{E}$ metabolism, ${ }^{4}$ abetalipoproteinemia, ${ }^{24}$ and $\mathrm{A}-\mathrm{T}^{5}$ were excluded by normal blood biochemistry results. Two other syndromes associated with defective DNA repair and neurodegeneration, xeroderma pigmentosum and Cockayne's syndrome, were excluded on the basis of clinical features. ${ }^{25}$

Symptoms of inherited metabolic disorders usually start in early infancy or childhood, and many of these disorders were previously considered to be limited to childhood. However, some of them should be considered in the differential diagnosis of adults. In these disorders, ataxia may be one symptom in addition to other CNS manifestations such as pyramidal symptoms, extrapyramidal symptoms, and dementia. In our patients, GM2 gangliosidosis, ${ }^{24}$ GM1 gangliosidosis, ${ }^{24}$ Gaucher disease, ${ }^{24}$ Krabbe disease, ${ }^{24}$ metachromatic leukodystrophy, ${ }^{24}$ and sialidosis ${ }^{26}$ were excluded by means of normal fibroblast enzyme assay results. Niemann-Pick disease type $\mathrm{C}$ was ruled out by normal bone marrow findings and normal morphology of visceral autopsy specimens. ${ }^{24}$ Urinary screening for glycosaminoglycans and oligosaccharides was also negative, ruling out mucopolysaccharidoses, disorders of glycoprotein degradation, and sialuria (Salla disease). ${ }^{27}$ Normal serum very long chain fatty acids and phytanic acid excluded adrenoleukodystrophy ${ }^{28}$ and Refsum disease. ${ }^{24}$ Wilson disease, disorders of amino acid metabolism, and cerebrotendinous xanthomatosis were excluded by biochemical results. ${ }^{24}$ No accumulation suggesting polyglucosan body disease was observed on sural nerve biopsy, ${ }^{29}$ and the adult form of ceroid lipofuscinosis does not fit well clinically because no lipopigment was observed in neurons at brain autopsy. ${ }^{30}$ Lack of increase in serum lactate, pyruvate, and cerebrospinal lactate levels, absence of ragged-red and cytochrome c oxidase-negative fibers on muscle biopsy, lack of known point mutations (MELAS, MERRF, NARP), and mtDNA deletions in muscle biopsy specimen make mitochondrial diseases unlikely. ${ }^{31}$ Autopsy findings are not compatible with typical Leigh's disease. ${ }^{32}$
Brain MRI findings in our patients are very characteristic, although not pathognomic. Unspecific white and gray matter abnormalities have been shown in many neurometabolic diseases such as lysosomal and peroxisomal storage disorders. ${ }^{33}$ MRI findings of mitochondrial diseases include leukodystrophic changes and deep gray nuclear changes in brainstem, thalamus, and basal ganglia, in addition to cerebral and cerebellar atrophy. ${ }^{34}$ Abnormal signal intensity of transverse pontine fibers has been reported in patients with SCA2 and other spinocerebellar ataxias. ${ }^{35-37}$ High-intensity signals have been detected on T2-weighted images of the brainstem and thalamus, in addition to diffuse, high-intensity areas in cerebral white matter, in some patients with DRPLA. ${ }^{38}$ MRI showed mild infratentorial atrophy and vague supratentorial atrophy.

With the exception of FRDA1, adult- and lateonset recessive ataxias are poorly described and understood. A new type was recently reported, ${ }^{39}$ but the disease was different in terms of conjunctival telangiectasias as a pathognomonic feature and elevated serum creatine kinase, gamma globulin, and $\alpha$-fetoprotein levels as abnormal laboratory findings. Two reports on testing large groups of patients with recessive or sporadic ataxia for the main mutation in the FRDA gene have recently been published. ${ }^{3,40} \mathrm{Mu}$ tant (GAA) $)_{n}$ expansion in FRDA was excluded in $25 \%$ and approximately $50 \%$ of the patients in both these studies. ${ }^{3,40}$

\section{Acknowledgment}

The authors thank all colleagues involved in the workup of the study participants, among others Vesa Juvonen, MSc, Professor Hannu Kalimo, Dr. Sanna-Kaisa, Dr. Toivakka-Hämäläinen, Kirsi Setälä, and Anne Eerola. The authors also thank T. Wise for technical assistance with mutational screening.

\section{References}

1. Harding AE. The hereditary ataxias and related disorders. Edinburgh: Churchill Livingstone, 1984.

2. Campuzano V, Montermini L, Moltò MD, et al. Friedreich's ataxia: autosomal recessive disease caused by an intronic GAA triplet repeat expansion. Science 1996;271:1423-1427.

3. Dürr A, Cossee M, Agid Y, et al. Clinical and genetic abnormalities in patients with Friedreich's ataxia. N Engl J Med 1996;335:1169-1175.

4. Ouachi K, Arita M, Kayden H, et al. Ataxia with isolated vitamin $\mathrm{E}$ deficiency is caused by mutations in alphatocopherol transfer protein. Nat Genet 1995;9:141-145.

5. Concannon P, Gatti RA. Diversity of ATM gene mutations detected in patients with ataxia-telangiectasia. Hum Mutat 1997;10:100-107.

6. Engert JC, Berube P, Mercier J, et al. ARSACS, a spastic ataxia common in northeastern Quebec, is caused by mutations in a new gene encoding an 11.5-kb ORF. Nat Genet 2000;24:120-125.

7. Mrissa N, Belal S, Ben Hamida C, et al. Linkage to chromosome 13q11-12 of an autosomal recessive cerebellar ataxia in a Tunisian family. Neurology 2000;54:1408-1414.

8. Klockgether T, Petersen D, Grodd W, Dichgans J. Early onset cerebellar ataxia with retained tendon reflexes: clinical, electrophysiological and MRI observations in comparison with Friedreich's ataxia. Brain 1991;114:1559-1573.

9. Geschwind DH, Perlman S, Grody WW, et al. Friedreich's ataxia GAA repeat expansion in patients with recessive or sporadic ataxia. Neurology 1997;49:1004-1009. 
10. Nikali K, Suomalainen A, Terwilligen J, Koskinen T, Weissenbach J, Peltonen L. Random search for shared chromosomal regions in four affected individuals: the assignment of a new hereditary ataxia locus. Am J Hum Genet 1995;56:10881095.

11. Bouchard JP, Richter A, Mathieu J, et al. Autosomal recessive spastic ataxia of Charlevoix-Saguenay. Neuromuscul Disord 1998;8:474-479.

12. Lindqvist A-KB, Magnusson PKE, Balciuniene J, et al. Chromosome-specific panels of tri- and tetranucleotide microsatellite markers for multiplex fluorescent detection of automated genotyping: evaluation of their utility in pathology and forensics. Genome Res 1996;6:1170-1176.

13. Orr HT, Chung MY, Banfi S, et al. Expansion of an unstable trinucleotide CAG repeat in spinocerebellar ataxia type 1 . Nat Genet 1993;4:221-226.

14. Pulst SM, Nechiporuk A, Nechiporuk T, et al. Moderate expansion of a normally biallelic trinucleotide repeat in spinocerebellar ataxia type 2. Nat Genet 1996;14:269-276.

15. Kawaguchi Y, Okamoto T, Taniwaki M, et al. CAG expansions in a novel gene for Machado-Joseph disease at chromosome 14q32.1. Nat Genet 1994;8:221-228.

16. Zhuchenko O, Bailey J, Bonnen P, et al. Autosomal dominant cerebellar ataxia (SCA 6) associated with small polyglutamine expansions in the alpha 1A-voltage-dependent calcium channel. Nat Genet 1997;15:62-69.

17. David G, Abbas N, Stevanin G, et al. Cloning of the SCA7 gene reveals a highly unstable CAG repeat expansion. Nat Genet 1997; 17:65-70.

18. Koob MD, Moseley ML, Schut LJ, et al. An untranslated CTG expansion causes a novel form of spinocerebellar ataxia (SCA8). Nat Genet 1999;21:379-384.

19. Matsuura T, Yamagata T, Burgess DL, et al. Large expansion of the ATTCT pentanucleotide repeat in spinocerebellar ataxia type 10 . Nat Genet 2000;26:191-194.

20. Holmes SE, O'Hearn EE, McInnis MG, et al. Expansion of a novel CAG trinucleotide repeat in the $5^{\prime}$ region of PPP2R2B is associated with SCA12. Nat Genet 1999;23:391-392.

21. Koide R, Ikeuchi T, Onodera O, et al. Unstable expansion of CAG repeat in hereditary dentatorubral-pallidoluysian atrophy (DRPLA). Nat Genet 1994;6:9-13.

22. Moraes CT, DiMauro S, Zeviani M, et al. Mitochondrial DNA deletions in progressive external ophthalmoplegia and Kearns-Sayre syndrome. N Engl J Med 1989;320:1293-1299.

23. Filla A, De Michele G, Cavalcanti F, et al. The relationship between trinucleotide (GAA) repeat length and clinical features in Friedreich ataxia. Am J Hum Genet 1996;59:554560.

24. Swanson PD. Diagnosis of inherited metabolic disorders affecting the nervous system. J Neurol Neurosurg Psychiatry 1995;59:460-470.
25. Chu G, Mayne L. Xeroderma pigmentosum, Cockayne syndrome and trichothiodystrophy: do the genes explain the diseases? Trends Genet 1996;12:187-192.

26. Johnson WG. Disorders of glycoprotein degradation: sialidosis, fucosidosis, alpha-mannosidosis, beta-mannosidosis, and aspartyl-glycosaminuria. In: Rosenberg RN, Prusiner SB, DiMauro S, Barchi R, eds. The molecular and genetic basic of neurological disease. Boston: Butterworth-Heineman, 1995: 335-369.

27. Romppanen J, Mononen I. Age-related reference values for urinary excretion of sialic acid and deoxysialic acid: application to diagnosis of storage disorders of free sialic acid. Clin Chem 1995;41:544-547.

28. Moser HW. Adrenoleukodystrophy: phenotype, genetics, pathogenesis and therapy. Brain 1997;120:1485-1508.

29. Bruno C, Servidei S, Shanske S, et al. Glycogen branching enzyme deficiency in adult polyglucosan body disease. Ann Neurol 1993;33:88-93.

30. Martin J-J. Adult type of neuronal ceroid-lipofuscinosis. J Inher Metab Dis 1993;16:237-240.

31. DiMauro S, Moraes CT. Mitochondrial encephalomyopathies. Arch Neurol 1993;50:1197-1208.

32. Chalmers RM, Lamont PJ, Nelson I, et al. A mitochondrial DNA tRNA(Val) point mutation associated with adult-onset Leigh syndrome. Neurology 1997;49:589-592.

33. Kendall BE. Disorders of lysosomes, peroxisomes, and mitochondria. Am J Neuroradiol 1992;13:621-653.

34. Wray SH, Provenzale JM, Johns DR, Thulborn KR. MR of the brain in mitochondrial myopathy. Am J Neuroradiol 1995;16: 1167-1173.

35. Giuffrida S, Saponara R, Restivo DA, et al. Supratentorial atrophy in spinocerebellar ataxia type 2: MRI study of 20 patients. J Neurol 1999;246:383-388.

36. Wüllner U, Klockgether T, Petersen D, Naegele T, Dichgans J. Magnetic resonance imaging in hereditary and idiopathic ataxia. Neurology 1993;43:318-325.

37. Murata Y, Yamaguchi S, Kawakami H, et al. Characteristic magnetic resonance imaging findings in Machado-Joseph disease. Arch Neurol 1998;55:33-37.

38. Koide R, Onodera O, Ikeuchi T, et al. Atrophy of the cerebellum and brainstem in dentatorubral pallidoluysian atrophy: influence of CAG repeat size on MRI findings. Neurology 1997;49:1605-1612.

39. Watanabe M, Sugai Y, Concannon P, et al. Familial spinocerebellar ataxia with cerebellar atrophy, peripheral neuropathy, and elevated level of serum creatine kinase, $\gamma$-globulin, and $\alpha$-fetoprotein. Ann Neurol 1998;44:265-269.

40. Pandolfo M. A reappraisal of the clinical features of Friedreich ataxia: what indications for a molecular test? Neurology 1999; 52(suppl 2):A260. Abstract. 